\title{
Prävalenz von SARS-CoV-2 bei Mitarbeitern eines Krankenhauses der Regel-|Schwerpunktversorgung in Nordrhein-Westfalen
}

\section{Prevalence of SARS-CoV-2 in employees of a general hospital in Northrhine-Westphalia, Germany}

Autoren

Martin Platten', Rita Cranen², Claudia Peters ${ }^{3}$, Hilmar Wisplinghoff1, 4, Albert Nienhaus ${ }^{3}, 5$, Alexander Daniel Bach ${ }^{6}$, Guido Michels ${ }^{7}$

\section{(ㄷ) (1) $\circledast \odot$}

Institute

1 Labor Dr. Wisplinghoff, Köln, Deutschland

2 Arbeitsmedizin, St.-Antonius-Hospital Eschweiler, Eschweiler, Deutschland

3 Kompetenzzentrum Epidemiologie und Versorgungsforschung bei Pflegeberufen (CVcare), Universitätsklinikum Hamburg-Eppendorf (UKE), Hamburg, Deutschland

4 Institut für Virologie und klinische Mikrobiologie, Universität Witten/Herdecke, Witten, Deutschland; Institut für medizinische Mikrobiologie, Immunologie und Hygiene, Universität zu Köln, Köln, Deutschland

5 Berufsgenossenschaft für Gesundheitsdienst und Wohlfahrtspflege (BGW), Abteilung Arbeitsmedizin, Gefahrstoffe und Gesundheitswissenschaften (AGG), Hamburg, Deutschland

6 Klinik für Plastische und Ästhetische Chirurgie, Handund Wiederherstellungschirurgie, Ärztlicher Direktor (in Vertretung für die Betriebsleitung), St.-Antonius-Hospital Eschweiler, Eschweiler, Deutschland

7 Klinik für Akut- und Notfallmedizin, St.-Antonius-Hospital Eschweiler, Eschweiler, Deutschland

Schlüsselwörter

Coronavirus, Pandemie, Querschnittsuntersuchung,

Prävalenzstudie, Arbeitsmedizin

Key words

Coronavirus, pandemic, cross-section analysis, prevalence analysis, occupational medicine

online publiziert 29.01 .2021

Bibliografie

Dtsch Med Wochenschr 2021; 146: e30-e38

DOI 10.1055/a-1322-5355

ISSN 0012-0472

(C) 2021. The Author(s).

This is an open access article published by Thieme under the terms of the Creative Commons Attribution-NonDerivative-NonCommercial License, permitting copying and reproduction so long as the original work is given appropriate credit. Contents may not be used for commecial purposes, or adapted, remixed, transformed or built upon. (https://creativecommons.org/licenses/by-nc-nd/4.0/)

Georg Thieme Verlag KG, Rüdigerstraße 14,

70469 Stuttgart, Germany
Korrespondenzadresse

Dr. Martin Platten

Labor Dr. Wisplinghoff, Horbeller Str. 18-20, 50858 Köln,

Deutschland

Tel.: +49/221/940505367

m.platten@wisplinghoff.de

Zusätzliches Material finden Sie unter

https://doi.org/10.1055/a-1322-5355

\section{ZUSAMMENFASSUNG}

Hintergrund Wir erhoben die Prävalenz von SARS-CoV-2 in der Belegschaft eines Krankenhauses der Regel-/Schwerpunktversorgung in Nordrhein-Westfalen im Rahmen einer Querschnittsstudie.

Methode Allen 1363 Mitarbeiter wurden ein nasopharyngealer Abstrich sowie eine Serologie auf SARS-CoV-2 angeboten. Zudem wurde ein Fragebogen zu Vorerkrankungen, Kontakten zu SARS-CoV-2-positiven Personen und COVID-19-typischen Symptomen vorgelegt.

Ergebnisse 1212 MitarbeiterInnen nahmen teil. 19 von 1363 $(1,4 \%)$ Mitarbeitern waren PCR-positiv (3 während der Studie, 16 vorher). Bei 40 (3,3\%) bzw. 105 (8,6\%) Mitarbeitern wurde IgG bzw. IgA nachgewiesen, bei 32 (2,6\%) IgG und IgA. Damit wurden insgesamt 47 Mitarbeiter positiv für SARS-CoV-2 getestet. In dieser Gruppe waren die häufigsten Symptome Kopfschmerzen (56\%), Müdigkeit (49\%), Halsschmerzen (49\%) und Husten (46\%), Fieber wurde in $33 \%$ berichtet. Positiv getestete Mitarbeiter gaben häufiger Kontakt zu einem COVID-19-Fall an (60,5\% vs. 37,3\%; p=0,006). Mitarbeiter mit isoliertem IgA-Nachweis gaben seltener Symptome an.

Schlussfolgerung 3,9\% der Mitarbeiter eines Krankenhauses der Regel-/Schwerpunktversorgung wurden zwischen dem 27.04. und 20.05.2020 positiv auf SARS-CoV-2 getestet. Der Anteil war geringer als erwartet; mögliche Gründe sind die geringe Durchseuchung der Bevölkerung und die umfangreichen, einheitlichen hausinternen Präventionsmaßnahmen.

\section{ABSTRACT}

Background We assessed the prevalence of SARS-CoV-2 in the staff of a general hospital in North-Rhine-Westphalia in a cross-sectional study. 
Method Employees ( $n=1363$ ) were offered a nasopharyngeal swab and serology for SARS-CoV-2. Additionally, employees completed a questionnaire about preexisting conditions, contacts with SARS-CoV-2-positive individuals and COVID-19specific symptoms.

Results 1212 employees participated. 19 of 1363 (1.4\%) employees tested positive by PCR ( 3 within and 16 before the study). 40 (3.3\%) and 105 (8.6\%) had IgG and IgA, respectively, 32 (2.6\%) both IgG and IgA. Overall, 47 employees tested positive. In this group, most frequently reported symptoms were headache $(56 \%)$, fatigue $(49 \%)$, sore throat (49\%), and cough ( $46 \%$ ); fever was reported by $33 \%$. SARS-CoV-2positive employees reported more frequently contact with COVID-19 cases (60.5\% vs. $37.3 \%, p=0.006)$. Employees testing positive only for IgA reported less symptoms.

Conclusion Between 27.04. and 20.05.2020, 3.9\% of the employees working in a general hospital were tested positive for SARS-CoV-2. This proportion was lower than expected; possible explanations are the low level of endemic infection and the extensive, uniform in-house preventative measures.

\section{Hintergrund}

Das neuartige Coronavirus Severe acute respiratory syndrome (SARS)-Coronavirus 2 (SARS-CoV-2) hat sich innerhalb kurzer Zeit weltweit ausgebreitet und stellt eine große Herausforderung für die Gesundheitssysteme der betroffenen Länder dar. Die Zahl der registrierten SARS-CoV-2-Fälle in Deutschland lag am 14. September 2020 bei 260355 , 9350 Patienten (3,6\%) mit SARS-CoV-2Infektion sind gestorben [1] (14. Januar: 1978590 Fälle, 43881 (2,2\%) sind gestorben). Bei Hospitalisation beträgt die Sterberate in Deutschland $22 \%$ [2].

Bislang gibt es mit Ausnahme des antiviralen Medikaments Remdesivir [3] keine spezifische Therapie und bis Ende Dezember 2020 keinen Impfstoff. Da ein signifikanter Anteil der SARS-CoV2-Infektionen oligo- oder asymptomatisch verläuft [4] und die räumliche Verbreitung eines pandemischen Erregers insbesondere zu Beginn der Pandemie nicht gleichmäßig ist, bleibt es unklar, in welchem Maße die Bevölkerung bereits infiziert wurde. Mitarbeiter im Gesundheitswesen haben aufgrund des häufigen und intensiven Kontakts zu Erkrankten ein besonders hohes Risiko, sich mit SARS-CoV-2 zu infizieren [5]. Daher ist es plausibel anzunehmen, dass die Prävalenz von SARS-CoV-2 bei Mitarbeitern im Gesundheitswesen höher ist als in der allgemeinen Bevölkerung.

Wir haben die Prävalenz von SARS-CoV-2 in der Belegschaft des St.-Antonius-Hospitals Eschweiler, eines Krankenhauses der Regel-/Schwerpunktversorgung in der Nähe von Aachen in Nordrhein-Westfalen mit 1363 Mitarbeitern und 443 Krankenhausbetten, im Rahmen einer Querschnittsstudie erhoben. Aufgrund der räumlichen Nähe zum Kreis Heinsberg, des in Deutschland ganz zu Beginn der Pandemie besonders betroffenen Landkreises, nahmen wir an, dass die Prävalenz von SARS-CoV-2 in dieser Population aufgrund frühzeitiger und intensiver Exposition gegenüber SARS-CoV-2-Patienten vergleichsweise hoch sein könnte. Zusätzlich haben wir mittels Fragebogens Symptome, Risikofaktoren und erinnerte Exposition gegenüber SARS-CoV-2 abgefragt.

\section{Material und Methoden}

Für diese Querschnittsuntersuchung wurden alle Mitarbeiter des St.-Antonius-Hospitals Eschweiler sämtlicher Bereiche im Rahmen der arbeitsmedizinischen Vorsorge zur Teilnahme eingeladen. Mit einem Anschreiben wurden die Mitarbeiter vorab über die Studienziele, den Studienablauf und den Datenschutz informiert (s. Online-Zusatzmaterial). Ausschlusskriterien gab es nicht. Die Untersuchung fand zwischen dem 27.04.2020 und 20.05.2020 statt. Die Teilnahme war freiwillig, und es gab keine Anreize, weder finanziell noch anderweitig. Alle Teilnehmer gaben ihr schriftliches Einverständnis zur Teilnahme an der Studie nach einer ausführlichen mündlichen und schriftlichen Aufklärung (s. Online-Zusatzmaterial). Die Finanzierung der Untersuchungen erfolgte über die Berufsgenossenschaft für Gesundheitsdienst und Wohlfahrtspflege Hamburg (Projektnummer: ext FF_1461). Die Studie wurde von der Ethikkommission der Ärztekammer Hamburg beraten (Ethikantrag Nr. PV7298); es wurden keine Bedenken gegen die Durchführung der Studie geäußert.

Die Teilnehmer füllten einen vom Studienteam entwickelten Fragebogen u. a. zu soziodemografischen Faktoren, Arbeitsbereich sowie Vorerkrankungen, Kontakten zu SARS-CoV-2-positiven Patienten oder Kollegen und COVID-19-typischen Symptomen aus (s. Online-Zusatzmaterial). Vorerkrankungen wurden aufgeteilt nach Organsystemen erhoben, typische Beispiele wurden im Fragebogen genannt (siehe Fragebogen SAH Mitarbeiter im Supplement). Bei allen Teilnehmern wurde ein tiefer nasopharyngealer Abstrich durchgeführt sowie eine Serumprobe abgenommen. Die nasopharyngealen Abstriche wurden mittels Reverse-Transkriptase-Polymerase-Kettenreaktion (RT-PCR) auf SARSCoV-2-RNA untersucht [6]. Die Serumproben wurden unter Verwendung eines kommerziell verfügbaren Enzyme Linked Immunosorbent Assays (ELISA) qualitativ auf SARS-CoV-2-Antikörper ( IgG und IgA) untersucht [7]. Alle Untersuchungen wurden gemäß den Anweisungen des Herstellers durchgeführt.

Für die Auswertung wurden diejenigen Mitarbeiter als positiv eingestuft, bei denen SARS-CoV-2-RNA mittels PCR und/oder lgG in der Serologie nachgewiesen wurde. Teilnehmer mit isoliertem Nachweis von IgA wurden wegen der unzureichenden Spezifität [8] nicht als positiv gewertet.

Die erhobenen Daten wurden mittels Nummerncode pseudonymisiert; eine Zuordnung der Daten zur Person war nur über eine bei der betreuenden Betriebsärztin elektronisch hinterlegte sicherheitsverschlüsselte Identifikationsliste möglich. Nach Beendigung der Studie werden alle Daten nach den gültigen Vorschriften für einen Zeitraum von 10 Jahren gespeichert und archiviert. Die im Rahmen der Studie abgenommenen Blutproben werden nach maximal 2 Jahren vernichtet. Alle Bestimmungen des Datenschutzes und der ärztlichen Schweigepflicht wurden eingehalten. 
Die Darstellung der Ergebnisse erfolgte für metrische Variablen durch Mittelwert (MW), Median, Standardabweichung (SD) sowie Spannweite (Range). Kategoriale Variablen wurden mit absoluten und relativen Häufigkeiten dargestellt. Gruppenunterschiede wurden durch Fishers exakten Test bei kategorialen Variablen und bei stetigen Variablen durch einen t-Test geprüft. Die Anteilswerte wurden mit $95 \%$-Konfidenzintervallen (95\%-KI) berechnet. Fehlende Angaben wurden berücksichtigt und in den Tabellen angegeben. Zur Ermittlung von „Kontakt mit einem positiven Fall“ als Risikofaktor wurde ein binär logistisches Regressionsmodell mit Korrektur nach Firth verwendet und Odds Ratios (OR) mit zugehörigen 95\%-KI berechnet. Dafür wurde ein positiver/negativer SARS-CoV-2-Befund der Mitarbeiter als abhängige Variable definiert. Die statistischen Analysen wurden mit den Programmen SPSS (Version 27, SPSS Inc.) und R (Version 4.0.3) durchgeführt.

\section{Ergebnisse}

Insgesamt 1212 von 1363 (88,9\%) Mitarbeitern im Alter von 1774 Jahren (Median 44 Jahre) nahmen an der Studie teil ( $>$ Abb. 1). Gründe für eine Nichtteilnahme waren vor allem Abwesenheit durch Urlaub oder Krankheit. - Tab. 1 zeigt die Charakteristiken der Studienpopulation. 951 (78,5\%) der Teilnehmer waren weiblich und 130 (10,7\%) waren älter als 59 Jahre, 240 (19,8\%) gaben an, aktuell zu rauchen. Der BMI lag im Median bei 25 . Die meisten Mitarbeiter waren in der unmittelbaren Patientenversorgung eingesetzt (865 von 1202 (78,5\%), keine Angabe von 110 Teilnehmern).

Allen 1212 Teilnehmern wurden tiefe nasopharyngeale Abstriche auf SARS-CoV-2-RNA entnommen. SARS-CoV-2-RNA war bei $3(0,2 \%)$ Teilnehmern nachweisbar. 16 Mitarbeiter waren bereits vor Beginn der Studie positiv auf SARS-CoV-2-RNA getestet worden, von denen 15 an der Studie teilnahmen. Somit wurden von Beginn der Pandemie bis zum 20.05.2020 insgesamt 19 von 1363 (1,4\%) Mitarbeiter positiv auf SARS-CoV-2-RNA getestet.

Auch die SARS-CoV-2-Serologie wurde bei allen 1212 Teilnehmern durchgeführt. Bei 40 (3,3\%) wurde IgG und bei 105 (8,6\%) IgA nachgewiesen, davon bei 32 (2,6\%) sowohl IgG als auch IgA. Bei 15 der 16 vor Beginn der Studie positiv auf SARS-CoV-2-RNA getesteten Mitarbeiter wurde eine Serologie durchgeführt, von denen bei 12 (80\%) Personen IgG nachweisbar war. Bei 2 der übrigen 3 Mitarbeiter wiederholten wir die Serologie im Verlauf, hier fand sich bei beiden weiterhin ein negatives IgG, ein Mitarbeiter wies ein positives IgA auf. Bei den 3 Teilnehmern mit positiver PCR im Studienzeitraum waren weder IgG noch IgA nachweisbar. Somit wurden insgesamt 47 Mitarbeiter positiv auf SARS-CoV-2 getestet (7 mittels PCR ohne positive Serologie und 40 mittels positiver Serologie, siehe $>$ Tab. 2).

In der Gruppe der positiv getesteten Mitarbeiter $(n=47)$ waren die häufigsten Symptome Kopfschmerzen (56\%), Müdigkeit/ Erschöpfung (49\%), Halsschmerzen (49\%) und Husten (46\%). Fieber wurde in $33 \%$ der Fälle berichtet ( $\triangleright$ Abb. 2).

414 von 1086 (38,1\%) Teilnehmern berichteten über mindestens einen Kontakt zu einer SARS-CoV-2-positiven Person; 282 von 403 $(70,0 \%)$ trugen zum Zeitpunkt des Kontakts einen Mund-NasenSchutz (MNS) und 133 von 390 (34,1\%) vollständige Schutzkleidung.

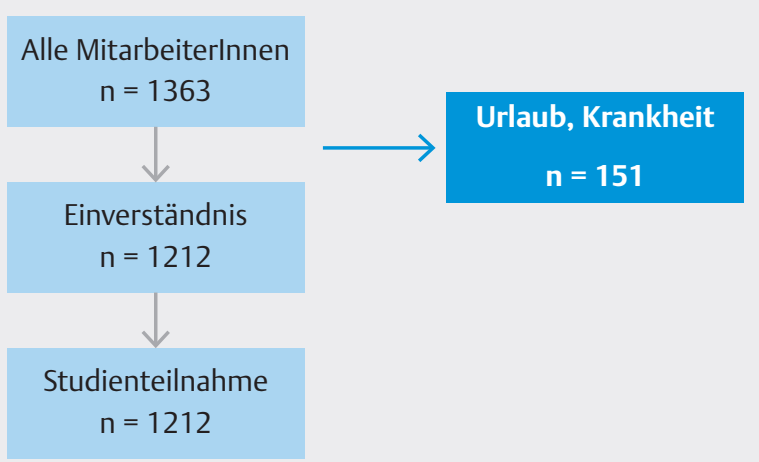

- Abb. 1 Studienteilnehmer.

- Tab. 1 Charakteristiken der Studienpopulation.

\begin{tabular}{|c|c|c|c|}
\hline Merkmal & & $\mathbf{N}$ & $\%$ \\
\hline Alter in Jahren & \multicolumn{3}{|c|}{$\begin{array}{l}\text { Mittelwert 43,1 (SD 13,1); Median 44,0; } \\
\text { Range 17-74 }\end{array}$} \\
\hline \multirow[t]{7}{*}{ Alter } & $<20$ Jahre & 18 & 1,5 \\
\hline & 20-29 Jahre & 227 & 18,7 \\
\hline & 30-39 Jahre & 253 & 20,9 \\
\hline & 40-49 Jahre & 251 & 20,7 \\
\hline & 50-59 Jahre & 332 & 27,4 \\
\hline & $\geq 60$ Jahre & 130 & 10,7 \\
\hline & keine Angabe & 1 & \\
\hline \multirow[t]{2}{*}{ Geschlecht } & männlich & 261 & 21,5 \\
\hline & weiblich & 951 & 78,5 \\
\hline BMI & \multicolumn{3}{|c|}{ Mittelwert 26,2 (SD 5,6); Median 25,1; Range 15-69 } \\
\hline \multirow{5}{*}{$\begin{array}{l}\text { BMI-Kategorien } \\
\text { (WHO-Einteilung) }\end{array}$} & $<18,5$ Untergewicht & 15 & 1,4 \\
\hline & $18,5-24,9$ Normalgewicht & 516 & 47,3 \\
\hline & 25,0-29,9 Übergewicht & 351 & 32,2 \\
\hline & $\geq 30,0$ Adipositas & 209 & 19,2 \\
\hline & keine Angabe & 121 & \\
\hline \multirow[t]{4}{*}{ Raucher } & nein & 682 & 63,4 \\
\hline & ja & 240 & 22,3 \\
\hline & ex & 153 & 14,2 \\
\hline & keine Angabe & 137 & \\
\hline \multirow{10}{*}{$\begin{array}{l}\text { Arbeitsbereich } \\
\text { Mehrfachnennung }\end{array}$} & Intensivstation & 151 & 13,7 \\
\hline & Internistische Notaufnahme & 75 & 6,8 \\
\hline & Chirurgische Notaufnahme & 42 & 3,8 \\
\hline & Isolationsstation & 67 & 6,1 \\
\hline & Normalstation & 452 & 41,0 \\
\hline & Onkologie & 54 & 4,9 \\
\hline & $\begin{array}{l}\text { nichtmedizinischer Bereich } \\
\text { ohne Patientenkontakt }\end{array}$ & 147 & 13,3 \\
\hline & $\begin{array}{l}\text { nichtmedizinischer Bereich } \\
\text { mit Patientenkontakt }\end{array}$ & 225 & 20,4 \\
\hline & sonstiger Bereich & 21 & \\
\hline & keine Angabe & 110 & 1,9 \\
\hline
\end{tabular}


- Tab. 2 Zusammensetzung der SARS-CoV-2-positiven Befunde.

\begin{tabular}{|l|l|l|l|l|}
\hline & IgG positiv & IgG grenzwertig & IgG negativ & GESAMT \\
\hline PCR positiv vor Studienzeitraum & 10 & 1 & 4 & 15 \\
\hline PCR positiv im Studienzeitraum & 0 & 0 & 3 & 3 \\
\hline PCR negativ & 21 & 8 & 1165 & 1194 \\
\hline GESAMT & 31 & 9 & 1172 & 1212 \\
\hline
\end{tabular}

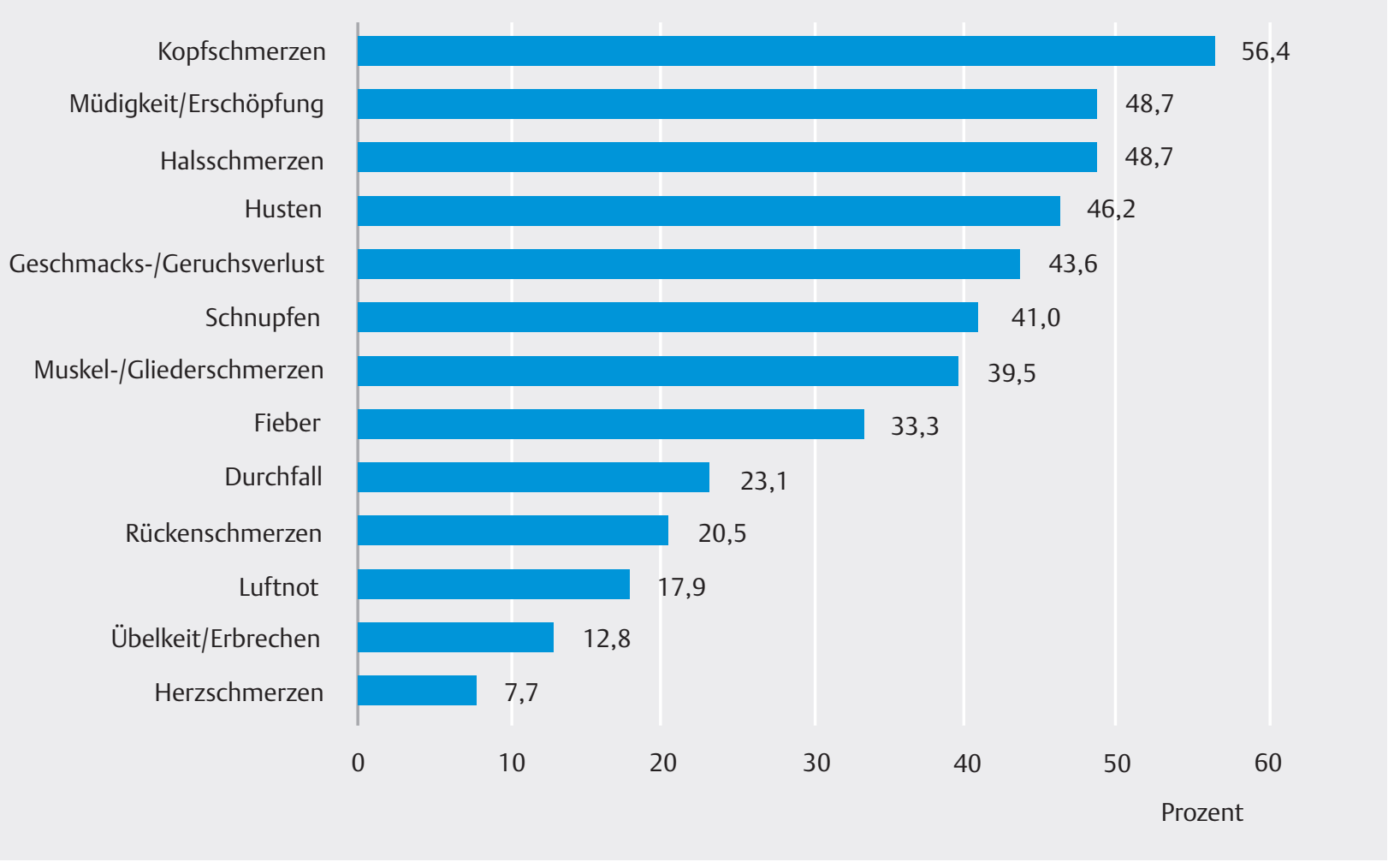

- Abb. 2 Symptome der positiv getesteten Mitarbeiter (PCR und/oder lgG).

293 von 405 (72,3\%) der Kontakte dauerten $\geq 15$ Minuten. 23 von 414 (5,6\%) der Mitarbeiter mit Kontakt zu SARS-CoV-2-Patienten wurden positiv auf SARS-CoV-2-RNA und/oder SARS-CoV-2-IgG getestet.

\section{Vergleich positiv getesteter (PCR und/oder IgG) mit negativ getesteten Mitarbeitern}

Wir stellten die Gruppe der positiv getesteten (PCR und/oder IgG, $n=47)$ den negativ getesteten Mitarbeitern $(n=1165)$ gegenüber ( $\triangleright$ Tab.3). Signifikante Unterschiede ergaben sich in einer Kategorie. So gaben positiv getestete Mitarbeiter häufiger an, Kontakt zu einem COVID-19-Fall gehabt zu haben (60,5\% vs. 37,3\%; $\mathrm{p}=0,006)$. Bei Mitarbeitern, die bei Kontakt mit einem COVID-19Fall einen Mund-Nasen-Schutz (MNS) trugen, den Kontakt mit einer Schutzausrüstung oder einen länger als 15 Minuten dauernden Kontakt hatten, zeigte sich kein deutlicher Unterschied zwischen den Gruppen. Keine Unterschiede ergaben sich bezüglich Alter,
BMI, Geschlecht, Rauchstatus und dem Vorhandensein von Vorerkrankungen. In der multivariablen Analyse zu den Kontaktkategorien ( $\triangleright$ Tab.4) zeigte sich ein erhöhtes Risiko für einen längeren Kontakt, der jedoch nicht statistisch signifikant war. Das Tragen eines MNS und das Tragen einer Schutzausrüstung beim Kontakt mit einem COVID-19-Fall hatten keinen Einfluss auf die Wahrscheinlichkeit, positiv getestet zu werden.

\section{Vergleich positiv getesteter (PCR und/oder IgG) mit isoliert für IgA positiv getesteten Mitarbeitern}

Zudem verglichen wir die Gruppe der positiv getesteten Mitarbeiter $(n=47)$ mit denjenigen, bei denen ausschließlich IgA nachgewiesen wurde $(n=73)$. Tab. 5 zeigt die Ergebnisse dieses Vergleichs. Hier ergaben sich statistisch signifikante Unterschiede in der Häufigkeit der Symptome bei positiver PCR und/oder IgG versus isoliert nachweisbarem IgA (67\% vs. $31 \%$; $p=<0,01)$; dies betraf die Häufigkeit von Kopfschmerzen $(p=0,01)$, Ge- 
- Tab. 3 Vergleich negativ vs. positiv (PCR und/oder IgG) getestete Mitarbeiter.

\begin{tabular}{|c|c|c|c|c|c|}
\hline Merkmal & \multicolumn{2}{|c|}{$\begin{array}{l}\text { Mitarbeiter, negativ } \\
\mathrm{n}=\mathbf{1 1 6 5}\end{array}$} & \multicolumn{2}{|c|}{$\begin{array}{l}\text { Mitarbeiter, positiv } \\
(\text { IgG+/PCR+) } \\
n=47\end{array}$} & p-Wert \\
\hline \multicolumn{6}{|l|}{ Alter (Jahre) } \\
\hline MW (SD) & \multicolumn{2}{|l|}{$43,3(13,1)$} & \multicolumn{2}{|l|}{$40,0(13,5)$} & 0,1 \\
\hline Median (Range) & \multicolumn{2}{|l|}{$44,0(17-74)$} & \multicolumn{2}{|l|}{$40,0(18-62)^{*}$} & \\
\hline \multicolumn{6}{|l|}{ BMI } \\
\hline $\mathrm{MW}$ (SD) & \multicolumn{2}{|l|}{$26,3(5,6)$} & \multicolumn{2}{|l|}{$25,3(5,8)$} & 0,3 \\
\hline \multirow[t]{2}{*}{ Median (Range) } & \multicolumn{2}{|l|}{$25,1(15-69)^{* *}$} & \multicolumn{2}{|l|}{$24,6(18-46)^{* * *}$} & \\
\hline & \% (95\%-KI) & Anzahl & \% (95\%-KI) & Anzahl & \\
\hline \multicolumn{6}{|l|}{ Geschlecht } \\
\hline männlich & $21,4(19,1-23,9)$ & 249/1165 & $25,5(14,4-40,6)$ & $12 / 47$ & 0,5 \\
\hline weiblich & $78,6(76,1-80,9)$ & $916 / 1165$ & $74,5(59,4-85,6)$ & $35 / 47$ & \\
\hline Raucher & $22,6(20,1-25,3)$ & $234 / 1037$ & $15,8(6,6-31,9)$ & $6 / 38$ & 0,4 \\
\hline \multicolumn{6}{|l|}{ Vorerkrankungen } \\
\hline Lunge & $12,2(10,3-14,3)$ & 129/1059 & $10,3(3,3-25,2)$ & $4 / 39$ & 1,0 \\
\hline Herz-Kreislauf & $19,2(16,9-21,7)$ & $203 / 1058$ & $12,8(4,8-28,2)$ & $5 / 39$ & 0,4 \\
\hline Niere & $1,4(0,8-2,4)$ & 15/1059 & $0,0(0,0-11,2)$ & $0 / 39$ & 1,0 \\
\hline onkologisch & $2,6(1,8-3,9)$ & $28 / 1059$ & $2,6(0,1-15,1)$ & $1 / 39$ & 1,0 \\
\hline Magen-Darm & $4,8(3,6-6,3)$ & $51 / 1059$ & $7,7(2,0-22,0)$ & $3 / 39$ & 0,4 \\
\hline Nervensystem & $4,2(3,2-5,7)$ & $45 / 1059$ & $0,0(0,0-11,2)$ & $0 / 39$ & 0,4 \\
\hline Stoffwechsel & $20,1(17,7-22,6)$ & 213/1061 & $10,3(3,3-25,2)$ & $4 / 39$ & 0,15 \\
\hline \multicolumn{6}{|c|}{ wissentlicher Kontakt mit SARS-CoV-2 } \\
\hline Kontakt mit positivem Fall & $37,3(34,4-40,3)$ & $391 / 1048$ & $60,5(43,5-75,5)$ & $23 / 38$ & 0,006 \\
\hline ...mit MNS & $25,8(23,2-28,5)$ & $270 / 1048$ & $28,9(16,0 ; 46,1)$ & $11 / 38$ & 0,7 \\
\hline ...mit Schutzausrüstung & $12,1(10,2-14,3)$ & $127 / 1048$ & $13,2(4,9-28,9)$ & $5 / 38$ & 0,8 \\
\hline$\ldots \geq 15 \min$ & $26,4(23,8-29,2)$ & $277 / 1048$ & $36,8(22,3-54,0)$ & $14 / 38$ & 0,2 \\
\hline MNS privat & $39,3(35,9-42,9)$ & $300 / 763$ & $51,6(33,4-69,4)$ & $16 / 31$ & 0,2 \\
\hline Indexperson bekannt & $71,4(30,3-94,9)$ & $5 / 7$ & $75,0(42,8-93,3)$ & $9 / 12$ & 1,0 \\
\hline $\begin{array}{l}\text { * keine Angabe (k. A.). } \\
\text { ** 113k. A. } \\
\text { ** } 8 \text { k. A. }\end{array}$ & & & & & \\
\hline
\end{tabular}

schmacks-/Geruchsverlust $(p<0,01)$, Schnupfen $(p=0,04)$, Muskel-/Gliederschmerzen $(p=0,008)$, Fieber $(p=0,003)$ und Diarrhö $(p=0,04)$. PCR- und/oder lgG-positive Mitarbeiter berichteten fast doppelt so häufig über Kontakte zu infektiösen Personen wie isoliert IgA-positive Mitarbeiter (61\% vs. $35 \%$; $p=0,02$ ). Es ergaben sich auch hier keine Unterschiede zwischen den Gruppen bezüglich Alter, BMI, Geschlecht, Rauchstatus und dem Vorhandensein von Vorerkrankungen.

\section{Diskussion}

Dies ist die erste Studie, in der das Infektionsgeschehen mit SARSCoV-2 im beruflichen Kontext bei Beschäftigten in einem deut- schen Krankenhaus mittels PCR und Serologie flächendeckend erhoben wurde. Insgesamt wurden 47 (3,9\%) Studienteilnehmer positiv auf SARS-CoV-2 getestet ( 7 nur mittels PCR und 40 mittels Serologie (lgG), von diesen 11 zusätzlich mittels PCR). Die häufigsten Symptome in dieser Gruppe waren Kopfschmerzen, Müdigkeit/Erschöpfung, Halsschmerzen und Husten. Positiv Getestete berichteten signifikant häufiger über Kontakt mit einem COVID-19-Fall.

\section{PCR}

Insgesamt wurden 19 von allen 1363 (1,4\%) Mitarbeitern des St.-Antonius-Krankenhauses Eschweiler bzw. 18 von den 1212 $(1,5 \%)$ Studienteilnehmern mittels PCR auf SARS-CoV-2 positiv 
- Tab. 4 Ergebnisse der logistischen Regression zum wissentlichen Kontakt mit einem positiven SARS-CoV-2-Fall im Vergleich von Mitarbeitern mit und ohne positiven Befund.

\begin{tabular}{|l|l|l|l|}
\hline & OR & $\mathbf{9 5} \%-\mathbf{K I}$ & $\mathbf{p}$-Wert \\
\hline Kontakt mit MNS & 0,78 & $0,27-2,05$ & 0,62 \\
\hline $\begin{array}{l}\text { Kontakt mit Schutz- } \\
\text { ausrüstung }\end{array}$ & 0,97 & $0,29-3,04$ & 0,96 \\
\hline Kontakt $\geq 15$ min & 1,81 & $0,77-4,05$ & 0,17 \\
\hline Alter & 0,98 & $0,96-1,01$ & 0,15 \\
\hline Geschlecht & 0,87 & $0,43-1,93$ & 0,72 \\
\hline
\end{tabular}

getestet, davon 3 (0,2\%) während des Untersuchungszeitraums vom 27.04.2020 bis zum 20.05.2020. Im Vergleich zu anderen bislang vorliegenden Erhebungen ist dies ein geringerer Anteil als erwartet. So lag der Anteil der Mitarbeiter mit positiver PCR auf SARS-CoV-2 in vergleichbaren Studien insgesamt zwischen 0,9 und $38 \%$, bei Screening-Studien an asymptomatischen Patienten zwischen 0,9 und 7,1\% [9-12]. Die relativ große Schwankungsbreite erklärt sich sicherlich durch Ort und Zeitraum der jeweiligen Studien. So wurde die höchste Prävalenz von 38\% durch Folgueira im März 2020 in Spanien ermittelt, in dem Monat, in dem der Höhepunkt der Epidemie in diesem Land zu verzeichnen war [11].

Es gibt viele mögliche Gründe für den vergleichsweise geringen Anteil positiver Nasopharyngealabstriche in der vorliegenden Untersuchung. So fand die Untersuchung in einem Zeitraum statt, in dem die Infektionsaktivität in Deutschland deutlich reduziert war, verdeutlicht anhand der Reproduktionszahl R, die bereits seit dem 21.03.2020 um oder unter 1 lag [13]. Schon seit mehreren Wochen bestehende Maßnahmen zur Verhinderung neuer Infektionen (Kontaktbeschränkungen, Schließung von Geschäften, Schulen und Kindergärten etc.) trugen mutmaßlich dazu bei. Wärmere Temperaturen im Rahmen der jahreszeitlichen Schwankungen können ebenfalls zu einer reduzierten Transmissionshäufigkeit beigetragen haben $[14,15]$, wie dies für die Influenza bekannt ist, die saisonal in den Wintermonaten der nördlichen bzw. südlichen Hemisphäre auftritt [16]. Die im Vergleich reduzierte Infektionsaktivität spiegelt sich auch darin wider, dass deutlich mehr Mitarbeiter vor Beginn unserer Untersuchung positiv auf SARS-CoV-2-RNA getestet wurden $(n=16)$ als im Rahmen der Studie $(n=3)$.

Zudem haben wahrscheinlich auch die hausintern getroffenen Maßnahmen am St.-Antonius-Krankenhaus Eschweiler zu einer niedrigen Infektionsrate unter der Belegschaft geführt. Das waren im Einzelnen:

- Rasche Etablierung, flächendeckende Umsetzung und strenge Überwachung der Hygieneregeln, in einem mittelgroßen

Krankenhaus vermutlich leichter umsetzbar als in großen Häusern; z. B. frühe Einführung der MNS-Pflicht für alle Mitarbeiter.

- Frühe Schaffung einer separierten COVID-19-Isolierstation, hier neben Isolation auch Kohortierung von Verdachtsfällen.
Somit bestanden von Beginn an 3 klar definierte Risikobereiche: Notaufnahme, Isolierstation und Intensivstation.

- Frühe Einrichtung einer Beratungsmöglichkeit für alle Mitarbeiter („Corona (-Sorgen)-Telefon“ mit Verfügbarkeit $24 \mathrm{~h}$ an 7 Tagen pro Woche) durch das Qualitätsmanagement und die Betriebsmedizin. In diesem Rahmen erfolgten neben psychologischer Betreuung und individueller Beratung nach Rücksprache mit der Betriebsmedizin auch eine Kontaktnachverfolgung und ein telefonisches Screening (Symptome, Kontakt-/ Indexperson, Aufenthalt in Risikogebieten).

- Individuelle betriebsmedizinische Betreuung mit Einrichtung einer hausinternen „Abstrichambulanz“ und Abstrichentnahme bei allen Verdachtsfällen mit Quarantäne, falls erforderlich; Versetzung von Schwangeren in Niedrigrisikobereiche (kein direkter Patientenkontakt, Homeoffice) und ggf. Arbeitsbefreiung; Forderung eines negativen Abstrichs nach Ende einer Quarantäne und bei Reiserückkehrern, was über die Anordnungen des lokalen Gesundheitsamtes hinausging.

- Frühzeitige und wiederholte Schulungen durch die Krankenhaushygiene mit einheitlichem und konsentiertem Inhalt zu den Themen Basishygiene und Umgang mit persönlicher Schutzausrüstung insbesondere in den Hochrisikobereichen Intensivstation, Notaufnahme und Isolierstation.

- Wöchentliche Online-Fortbildung (Schaffung eines Sammelaccounts für alle Mitarbeiter) und Information aller Mitarbeiter mittels Newsletter (Entwicklung der internationalen und nationalen Fallzahlen, aktuelle Studien, aktuelle Empfehlungen des Robert-Koch-Instituts, regionale Empfehlungen und hausinterne Beschlüsse des Krisenstabs).

Zuletzt ist es nicht auszuschließen, dass es durch eine fehlerhafte Entnahme oder Verarbeitung der Nasopharyngealabstriche zu falsch negativen Ergebnissen der PCR gekommen sein kann. Allerdings gibt es Hinweise darauf, dass Nasopharyngealabstriche zur Diagnose einer SARS-CoV-2-Infektion zumindest im Vergleich zu oropharyngealen Abstrichen sensitiver sind [17]. Zudem ist bekannt, dass ein Abstrich der oberen Atemwege negativ ausfallen kann, insbesondere bei schweren Verläufen mit SARS-CoV-2Pneumonie [18]. Die höchste SARS-CoV-2-Konzentration in den oberen Atemwegen findet sich mehrheitlich in der Zeit kurz vor bis nach Symptombeginn $[19,20]$.

\section{Serologie}

Bei 40 (3,3\%) Mitarbeitern wurde IgG gegen SARS-CoV-2 nachgewiesen, bei 27 (2,2\%) sowohl IgG als auch IgA. Bei 107 (8,8\%) Mitarbeitern war IgA nachzuweisen. In einer Studie an 316 Mitarbeitern der Uniklinik Essen wurde zwischen dem 25.03.2020 und 21.04.2020 in 5 (1,6\%) Fällen IgG nachgewiesen [21]. Der niedrigere Anteil im Vergleich zu den von uns erhobenen Daten ist wahrscheinlich darauf zurückzuführen, dass die Untersuchung zu einem früheren Zeitpunkt in der Pandemie stattfand. In einer weiteren Studie in Spanien fand sich bei 578 Mitarbeitern der Universitätsklinik Barcelona zwischen dem 28.03.2020 und 09.04.2020 bei 44 (7,6\%) IgG gegen SARS-CoV-2 [12]. Zur besseren Einordnung der vorliegenden Ergebnisse sind weitere Erhebungen wünschenswert. 
- Tab. 5 Vergleich positiv (PCR und/oder IgG) getestete Mitarbeiter vs. isolierter IgA-Nachweis.

\begin{tabular}{|c|c|c|c|c|c|}
\hline Merkmal & \multicolumn{2}{|l|}{$\begin{array}{l}\text { Mitarbeiter } \lg A+ \\
\mathrm{n}=73\end{array}$} & \multicolumn{2}{|c|}{$\begin{array}{l}\text { Mitarbeiter } \mathrm{lgG}+/ \mathrm{PCR}+ \\
\mathrm{n}=47\end{array}$} & p-Wert \\
\hline \multicolumn{6}{|l|}{ Alter (Jahre) } \\
\hline $\mathrm{MW}(\mathrm{SD})$ & \multicolumn{2}{|l|}{$42,0(13,1)$} & \multicolumn{2}{|l|}{$40,0(13,5)$} & 0,4 \\
\hline Median (Range) & \multicolumn{2}{|l|}{$44,0(19-71)$} & \multicolumn{2}{|l|}{$40,0(18-62)^{*}$} & \\
\hline \multicolumn{6}{|l|}{ BMI } \\
\hline $\mathrm{MW}(\mathrm{SD})$ & \multicolumn{2}{|l|}{$26,1(5,9$} & \multicolumn{2}{|l|}{$25,3(5,8)$} & 0,5 \\
\hline \multirow[t]{2}{*}{ Median (Range) } & \multicolumn{2}{|l|}{$24,5(18-52)^{* *}$} & \multicolumn{2}{|l|}{$24,6(18-46)^{* * *}$} & \\
\hline & (95\%-KI) & Anzahl & (95\%-KI) & Anzahl & \\
\hline \multicolumn{6}{|l|}{ Geschlecht } \\
\hline männlich & $37,0(26,2-49,1)$ & 27 & $25,5(14,4-40,6)$ & 12 & 0,2 \\
\hline weiblich & $63,0(50,9-73,8)$ & 46 & $74,5(59,4-85,6)$ & 35 & \\
\hline Raucher & $12,3(5,8-23,4)$ & $8 / 65$ & $15,8(6,6-31,9)$ & $6 / 38$ & 0,8 \\
\hline \multicolumn{6}{|l|}{ Vorerkrankungen } \\
\hline Lunge & $12,1(5,7-23,0)$ & $8 / 66$ & $10,3(3,3-25,2)$ & $4 / 39$ & 1,0 \\
\hline Herz-Kreislauf & $15,2(7,9-26,6)$ & $10 / 66$ & $12,8(4,8-28,2)$ & $5 / 39$ & 1,0 \\
\hline Niere & $0,0(0,0-6,9)$ & $0 / 66$ & $0,0(0,0-11,2)$ & $0 / 39$ & 1,0 \\
\hline onkologisch & $3,0(0,5-11,5)$ & $2 / 66$ & $2,6(0,1-15,1)$ & $1 / 39$ & 1,0 \\
\hline Magen-Darm & $6,1(2,0-15,6)$ & $4 / 66$ & $7,7(2,0-22,0)$ & $3 / 39$ & 0,7 \\
\hline Nervensystem & $1,5(0,1-9,3)$ & $1 / 66$ & $0,0(0,0-11,2)$ & $0 / 39$ & 1,0 \\
\hline Stoffwechsel & $12,1(5,7-23,0)$ & $8 / 66$ & $10,3(3,3-25,2)$ & $4 / 39$ & 1,0 \\
\hline \multicolumn{6}{|l|}{ Symptome } \\
\hline Symptome & $30,8(20,2-43,6)$ & $20 / 65$ & $66,7(49,7-80,4)$ & $26 / 39$ & $<0,01$ \\
\hline Kopfschmerzen & $30,3(19,9-43,0)$ & $20 / 66$ & $56,4(39,8-71,8)$ & $22 / 39$ & 0,01 \\
\hline Müdigkeit/Erschöpfung & $30,3(19,9-43,0)$ & $20 / 66$ & $48,7(32,7-65,0)$ & $19 / 39$ & 0,07 \\
\hline Halsschmerzen & $28,8(18,6-41,4)$ & $19 / 66$ & $48,7(32,7-65,0)$ & 19/39 & 0,06 \\
\hline Husten & $28,8(18,6-41,4)$ & $19 / 66$ & $46,2(30,4-62,6)$ & $18 / 39$ & 0,09 \\
\hline $\begin{array}{l}\text { Geschmacks-/Geruchsver- } \\
\text { lust }\end{array}$ & $3,0(0,5-11,5)$ & $2 / 66$ & $43,6(28,2-60,2)$ & $17 / 39$ & $<0,01$ \\
\hline Schnupfen & $21,2(12,5-33,3)$ & $14 / 66$ & $41,0(26,0-57,8)$ & $16 / 39$ & 0,04 \\
\hline Muskel-/Gliederschmerzen & $15,2(7,9-26,6)$ & $10 / 66$ & $39,5(24,5-56,5)$ & $15 / 38$ & 0,008 \\
\hline Fieber & $9,1(3,7-19,4)$ & $6 / 66$ & $33,3(19,6-50,3)$ & $13 / 39$ & 0,003 \\
\hline Durchfall & $7,6(2,8-17,5)$ & $5 / 66$ & $23,1(11,7-39,7)$ & $9 / 39$ & 0,04 \\
\hline Rückenschmerzen & $18,5(10,3-30,4)$ & $12 / 65$ & $20,5(9,9-36,9)$ & $8 / 39$ & 0,8 \\
\hline Luftnot & $6,1(2,0-15,6)$ & $4 / 66$ & $17,9(8,1-34,1)$ & $7 / 39$ & 0,1 \\
\hline Übelkeit/Erbrechen & $6,1(2,0-15,6)$ & $4 / 66$ & $12,8(4,8-28,2)$ & $5 / 39$ & 0,3 \\
\hline Herzschmerzen & $4,5(1,2-13,6)$ & $3 / 66$ & $7,7(2,0-22,0)$ & $3 / 39$ & 0,7 \\
\hline \multicolumn{6}{|c|}{ wissentlicher Kontakt mit SARS-CoV-2 } \\
\hline Kontakt mit positivem Fall & $35,4(24,2-48,3)$ & $23 / 65$ & $60,5(43,5-75,5)$ & $23 / 38$ & 0,02 \\
\hline ...mit MNS & $15,4(8,0-26,9)$ & $10 / 65$ & $28,9(16,0-46,1)$ & $11 / 38$ & 0,1 \\
\hline ...mit Schutzausrüstung & $6,2(2,0-15,8)$ & $4 / 65$ & $13,2(4,9-28,9)$ & $5 / 38$ & 0,3 \\
\hline$\ldots \geq 15 \min$ & $26,2(16,4-38,8)$ & $17 / 65$ & $36,8(22,3-54,0)$ & $14 / 38$ & 0,3 \\
\hline MNS privat & $41,7(27,9-56,7)$ & $20 / 48$ & $51,6(33,4-69,4)$ & $16 / 31$ & 0,5 \\
\hline
\end{tabular}


Der Stellenwert der Serologie bei SARS-CoV-2 ist noch unklar. Dies betrifft Sensitivität und Spezifität ebenso wie den Zeitraum der Nachweisbarkeit spezifischer Antikörper nach Infektion [22, 23]. Die Spezifität von IgA für SARS-CoV-2 ist gering [8, 24], und es wird diskutiert, dass ein isolierter Nachweis von IgA eine Kreuzreaktion auf andere Coronaviren, welche in der Population zirkulieren und banale Erkältungen auslösen, darstellt [25].

Es gibt Hinweise, dass asymptomatisch Infizierte seltener eine messbare Antikörperantwort entwickeln [26]. Zudem können falsch positive Ergebnisse im Rahmen einer Kreuzreaktivität der eingesetzten Serologie auf andere Coronaviren vorkommen [25]. Es existieren zwar Hinweise, dass der Nachweis von Antikörpern gegen SARS-CoV-2 mit einer Immunität einhergeht [27], über Dauer und Robustheit einer Immunität besteht aber noch Unklarheit [28].

Im Vergleich zu positiv Getesteten (PCR und/oder lgG) berichteten Mitarbeiter mit isoliertem IgA-Nachweis signifikant seltener COVID-19-typische Symptome. Obgleich die Gruppengrößen relativ klein waren, unterstützen diese Ergebnisse die Beobachtung, dass ein isolierter IgA-Nachweis die Diagnose von COVID-19 nicht rechtfertigt.

\section{Limitationen und Stärken}

Die vorliegende Studie hat einige Limitationen. So ergab sich bei lediglich 47 von 1212 Mitarbeitern anhand von PCR oder Serologie ein relativ sicherer Anhalt für eine SARS-CoV-2-Infektion, sodass weiterführende Zusammenhangsanalysen nicht möglich waren. Aufgrund der kleinen Fallzahlen ist es wahrscheinlich, dass bestehende Zusammenhänge nicht detektiert werden konnten.

Mitarbeiter, die bei Kontakt mit einem COVID-19-Fall einen MNS trugen, wurden zwar häufiger negativ getestet, aber ein statistisch begründeter, protektiver Effekt kann mit dieser Untersuchung nicht gegeben werden. Andere Studien dagegen geben Hinweise, dass das Tragen eines MNS Infektionen mit SARS-CoV-2 verhindern kann [29, 30].

Die hohe Responserate und somit die beinahe vollständige Erfassung aller Mitarbeiter eines Krankenhauses erlauben eine gute Beschreibung des Infektionsgeschehens bei den Beschäftigten.

\section{Ausblick und Fazit}

Wir planen Wiederholungsuntersuchungen nach 3, 6 und 12 Monaten, um weitere Erkenntnisse über die Verbreitung von SARSCoV-2 und die Dynamik der Antikörperantwort bei den Beschäftigten über den Zeitverlauf zu gewinnen.

3,9\% der Mitarbeiter eines Krankenhauses der Regel-/Schwerpunktversorgung wurden im Zeitraum vom 27.04.2020 bis 20.05.2020 mittels PCR und/oder Serologie positiv auf SARSCoV-2 getestet. Der Anteil war geringer als erwartet; mögliche Gründe finden sich neben der noch relativ geringen Durchseuchung der Bevölkerung in den umfangreichen, einheitlichen hausinternen Präventionsmaßnahmen. Im Rahmen der geplanten Wiederholungsuntersuchungen rechnen wir mit einem Anstieg positiv Getesteter. Mitarbeiter mit isoliertem IgA-Nachweis gaben signifikant seltener COVID-19-typische Symptome an - dies unterstützt die Hinweise, dass ein isolierter IgA-Nachweis die Diagnose von COVID-19 nicht rechtfertigt.

\section{KERNAUSSAGEN}

- 3,9\% der Mitarbeiter eines Krankenhauses der Regel- 1 Schwerpunktversorgung wurden positiv auf SARS-CoV-2 getestet.

- Die häufigsten genannten Symptome waren Kopfschmerzen (56\%), Müdigkeit (49\%), Halsschmerzen (49\%) und Husten (46\%); Fieber wurde in $33 \%$ berichtet.

- Positiv getestete Mitarbeiter gaben signifikant häufiger Kontakt zu einem COVID-19-Fall an (60,5\% vs. 37,3\%; $\mathrm{p}=0,006)$.

- Mitarbeiter mit isoliertem IgA-Nachweis gaben seltener COVID-19-typische Symptome an.

Interessenkonflikt

Die Autorinnen/Autoren geben an, dass kein Interessenkonflikt besteht.

Danksagung

Die Studie wurde in Teilen von der Berufsgenossenschaft für Gesundheitsdienst und Wohlfahrtspflege in Hamburg finanziert. Wir danken dem Studienteam des St.-Antonius-Hospitals Eschweiler: Astrid Lichtenstein, Ines Martinett, Astrid Engels, Christina Kexel, Beate Metterhausen, Sabrina Hermanns, Anna-Maria Kurth, Wioletta Osko, Monika Beumers, Dr. Kathrin Bellen, Dr. Gabriele Heller, Dr. Torsten Rütters, Benjamin Bruno und vielen weiteren Personen, zudem dem Laborteam im Labor Dr. Wisplinghoff: Dr. Roger Grosser, Dr. Lars Rivaletto, Dr. Dirk Happich, Dr. Fabian Wisplinghoff, Dr. Angela Nowag, Ute Pöppelmann, Dr. Melanie Polke. Zudem danken wir den Mitarbeitern des St.-Antonius-Hospitals Eschweiler für die Teilnahme an der Studie.

\section{Literatur}

[1] Robert-Koch-Institut. COVID-19: Fallzahlen in Deutschland und weltweit. In 2020 https://www.rki.de/DE/Content/InfAZ/N/Neuartiges_Coronavirus/ Fallzahlen.html

[2] Karagiannidis C, Mostert C, Hentschker C et al. Case characteristics, resource use, and outcomes of 10021 patients with COVID-19 admitted to 920 German hospitals: an observational study. Lancet Respir Med 2020; 8: 853-862

[3] Beigel JH, Tomashek KM, Dodd LE et al. Remdesivir for the Treatment of Covid-19 - Preliminary Report. N Engl J Med 2020. doi:10.1056/ NEJMoa2007764

[4] Nishiura H, Kobayashi T, Suzuki A et al. Estimation of the asymptomatic ratio of novel coronavirus infections (COVID-19). Int J Infect Dis 2020. doi:10.1016/j.ijid.2020.03.020

[5] Koh D. Occupational risks for COVID-19 infection. Occup Med (Lond) 2020; 70: 3-5

[6] Corman VM, Landt O, Kaiser M et al. Detection of 2019 novel coronavirus (2019-nCoV) by real-time RT-PCR. Euro Surveill 2020; 25 (3): 2000045

[7] Euroimmun. Characteristics of EUROIMMUN ELISA for COVID-19 diagnostics. In 2020

[8] Van Elslande J, Houben E, Depypere M et al. Diagnostic performance of seven rapid $\mathrm{IgG} / \mathrm{lgM}$ antibody tests and the Euroimmun $\mathrm{IgA} / \mathrm{lgG}$ ELISA in COVID-19 patients. Clin Microbiol Infect 2020; 26: 1082-1087 
[9] Reusken CB, Buiting A, Bleeker-Rovers C et al. Rapid assessment of regional SARS-CoV-2 community transmission through a convenience sample of healthcare workers, the Netherlands, March 2020. Euro Surveill 2020; 25 (12): 2000334

[10] Schwierzeck V, Correa-Martinez CL, Schneider KN et al. SARS-CoV-2 in the Employees of a Large University Hospital. Dtsch Arztebl Int 2020; 117: 344-345

[11] Folgueira MD, Munoz-Ruiperez C, Alonso-Lopez MA et al. SARS-CoV-2 infection in Health Care Workers in a large public hospital in Madrid, Spain, during March 2020. medRxiv 2020. doi:10.1101/ 2020.04.07.20055723:2020.2004.2007.20055723

[12] Garcia-Basteiro AL, Moncunill G, Tortajada M et al. Seroprevalence of antibodies against SARS-CoV-2 among health care workers in a large Spanish reference hospital. Nat Commun 2020; 11: 3500

[13] Robert-Koch-Institut. Nowcasting und R-Schätzung: Schätzung der aktuellen Entwicklung der SARS-CoV-2-Epidemie in Deutschland. In 2020 https://www.rki.de/DE/Content/InfAZ/N/Neuartiges_Coronavirus/ Projekte_RKI/Nowcasting.html

[14] Sobral MFF, Duarte GB, da Penha Sobral AIG et al. Association between climate variables and global transmission oF SARS-CoV-2. Sci Total Environ 2020; 729: 138997

[15] Liu J, Zhou J, Yao J et al. Impact of meteorological factors on the COVID-19 transmission: A multi-city study in China. Sci Total Environ 2020; 726 : 138513

[16] Fuhrmann C. The Effects of Weather and Climate on the Seasonality of Influenza: What We Know and What We Need to Know. Geography Compass 2010; 4: 718-730

[17] Wang X, Tan L, Wang $X$ et al. Comparison of nasopharyngeal and oropharyngeal swabs for SARS-CoV-2 detection in 353 patients received tests with both specimens simultaneously. Int J Infect Dis 2020; 94: 107-109

[18] Huang Y, Chen S, Yang Z et al. SARS-CoV-2 Viral Load in Clinical Samples of Critically III Patients. Am J Respir Crit Care Med 2020. doi:10.1164/ rccm.202003-0572LE

[19] To KK, Tsang OT, Leung WS et al. Temporal profiles of viral load in posterior oropharyngeal saliva samples and serum antibody responses during infection by SARS-CoV-2: an observational cohort study. Lancet Infect Dis 2020; 20: 565-574

[20] Zou L, Ruan F, Huang M et al. SARS-CoV-2 Viral Load in Upper Respiratory Specimens of Infected Patients. N Engl J Med 2020; 382: 11771179

[21] Korth ], Wilde B, Dolff S et al. SARS-CoV-2-specific antibody detection in healthcare workers in Germany with direct contact to COVID-19 patients. J Clin Virol 2020; 128: 104437

[22] Zhao J, Yuan Q, Wang $\mathrm{H}$ et al. Antibody responses to SARS-CoV-2 in patients of novel coronavirus disease 2019. Clin Infect Dis 2020. doi:10.1093/cid/ciaa344

[23] Jin Y, Wang M, Zuo Z et al. Diagnostic value and dynamic variance of serum antibody in coronavirus disease 2019. Int J Infect Dis 2020; 94: 49-52

[24] Euroimmun. Anti-SARS-CoV-2 ELISA (IgA). In 2020 https://www.corona virus-diagnostik.de/documents/Indications/Infections/Coronavirus/ El_2606_D_DE_B.pdf

[25] Meyer B, Drosten C, Muller MA. Serological assays for emerging coronaviruses: challenges and pitfalls. Virus Res 2014; 194: 175-183

[26] Yongchen Z, Shen H, Wang X et al. Different longitudinal patterns of nucleic acid and serology testing results based on disease severity of COVID-19 patients. Emerg Microbes Infect 2020; 9: 833-836

[27] Ju B, Zhang Q, Ge J et al. Human neutralizing antibodies elicited by SARS-CoV-2 infection. Nature 2020. doi:10.1038/s41586-020-2380-z

[28] Robert-Koch-Institut. SARS-CoV-2 Steckbrief zur Coronavirus-Krankheit2019 (COVID-19). In 2020 https://www.rki.de/DE/Content/InfAZ/N/ Neuartiges_Coronavirus/Steckbrief.html;jsessionid=A9C2852F363 A25816D60222D96FA660C.internet121?nn=2386228

[29] Leung NHL, Chu DKW, Shiu EYC et al. Respiratory virus shedding in exhaled breath and efficacy of face masks. Nat Med 2020; 26: 676-680

[30] Cheng KK, Lam TH, Leung CC. Wearing face masks in the community during the COVID-19 pandemic: altruism and solidarity. Lancet 2020. doi:10.1016/S0140-6736(20)30918-1 